\title{
A FIXED POINT THEOREM FOR MULTIVALUED NONEXPANSIVE MAPPINGS IN A UNIFORMLY CONVEX BANACH SPACE
}

\author{
BY TECK-CHEONG LIM ${ }^{1}$
}

Communicated by Alberto Calderón, February 19, 1974

Let $C$ be a nonempty weakly compact convex subset of a Banach space $X$, and $\mathscr{C}(C)$ be the family of nonempty compact subsets of $C$ equipped with the Hausdorff metric. Let $T: C \rightarrow \mathscr{C}(C)$ be a nonexpansive mapping, i.e. for each $x, y \in C$,

$$
H(T(x), T(y)) \leqq\|x-y\|,
$$

where $H(A, B)$ denotes the Hausdorff distance between $A$ and $B$. A point $x \in C$ is called a fixed point of $T$ if $x \in T x$. Fixed point theorems for such mappings $T$ have been established by Markin [11] for Hilbert spaces, by Browder [2] for spaces having weakly continuous duality mapping, and by Lami Dozo [7] for spaces satisfying Opial's condition. Lami Dozo's result is also generalized by Assad and Kirk [1]. By making use of Edelstein's asymptotic center [4], [5], we are able to prove Theorem 1 . Let $C$ be a closed convex subset of a uniformly convex Banach space and let $\left\{u_{i}\right\}$ be a bounded sequence in $C$. The asymptotic center $x$ of $\left\{u_{i}\right\}$ in (or with respect to) $C$ is the unique point in $C$ such that

$$
\lim \sup _{i}\left\|x-u_{i}\right\|=\inf \left\{\limsup _{i}\left\|y-u_{i}\right\|: y \in C\right\} .
$$

The number $r=\inf \left\{\lim \sup _{i}\left\|y-u_{i}\right\|: y \in C\right\}$ is called the asymptotic radius of $\left\{u_{i}\right\}$ in $C$. Existence of the unique asymptotic center is proved by Edelstein in [5]. Results on ordinal numbers used here may be found in [13].

THEOREM 1. Let $X$ be a uniformly convex Banach space and $C$ be a closed convex bounded nonempty subset of $X$. Let $T: C \rightarrow \mathscr{C}(C)$ be a nonexpansive mapping from $C$ into the family of nonempty compact subsets of

AMS (MOS) subject classifications (1970). Primary 46A05.

Key words and phrases. Fixed point, multivalued nonexpansive mapping, uniformly convex Banach space, asymptotic center.

1 This research was conducted while the author held an Izzak Walton Killam Memorial Scholarship under the supervision of Professor Michael Edelstein. 
$C$ (equipped with the Hausdorff metric). Then $T$ has a fixed point, i.e. there exists $x \in C$ with $x \in T x$.

Proof. Let $a$ be a point in $C$ fixed throughout the proof. Let $\left\{\lambda_{m}\right\}$ be a decreasing sequence of positive numbers and $\lim \lambda_{m}=0$. For each $m$, the mapping $T_{m}: C \rightarrow \mathscr{C}(C)$ defined by $T_{m}(x)=\lambda_{m} a+\left(1-\lambda_{m}\right) T x$ is a contraction mapping and hence has a fixed point $x_{m}$ (Nadler [12]). Thus $x_{m} \in \lambda_{m} a+\left(1-\lambda_{m}\right) T x_{m}$, and there exists $y_{m} \in T x_{m}$ with $x_{m}=\lambda_{m} a+$ $\left(1-\lambda_{m}\right) y_{m}$. Since $C$ is bounded, we have

$$
\left\|x_{m}-y_{m}\right\|=\lambda_{m}\left\|a-\dot{y_{m}}\right\| \rightarrow 0 \quad \text { as } m \rightarrow \infty .
$$

To facilitate the later description, we define $i:\left\{x_{m}\right\} \rightarrow\left\{y_{m}\right\}$ by $i\left(x_{m}\right)=y_{m}$ for all $m$. We say that a sequence $\left\{x_{n}\right\}$ is an essential subsequence of $\left\{y_{m}\right\}$ if for some $N>0,\left\{x_{n}\right\}_{n \geqq N}$ is a subsequence of $\left\{y_{m}\right\}$.

Define the sequence $\left\{x_{m}^{(0)}\right\}$ to be $\left\{x_{m}\right\}$, i.e. $x_{m}^{(0)}=x_{m}$ for each $m \geqq 1$. Let $\Omega$ be the first uncountable ordinal and $\beta$ be a countable ordinal, i.e. $\beta<\Omega$. Suppose that $\left\{x_{m}^{(\alpha)}\right\}$ has been defined for every ordinal $\alpha$ less than $\beta$ in such a way that $\left\{x_{m}^{(\gamma)}\right\}$ is an essential subsequence of $\left\{x_{m}^{(\delta)}\right\}$ whenever $\delta<\gamma<\beta$. We define $\left\{x_{m}^{(\beta)}\right\}$ as follows:

Case 1. $\beta$ has an immediate predecessor, i.e., $\beta=\alpha+1$ for some $\alpha<\Omega$. Let $z_{\alpha}$ be the asymptotic center of $\left\{x_{m}^{(\alpha)}\right\}$ in $C$. For each $m$, let $p_{m} \in T z_{\alpha}$ be chosen such that

$$
\left\|p_{m}-y_{m}^{(\alpha)}\right\| \leqq\left\|z_{\alpha}-x_{m}^{(\alpha)}\right\|,
$$

where $y_{m}^{(\alpha)}=i\left(x_{m}^{(\alpha)}\right)$; existence of such a $p_{m}$ is a consequence of the nonexpansiveness of $T$ and the compactness of $T z_{\alpha}$. Since $T z_{\alpha}$ is compact and $\left\{p_{m}\right\} \subseteq T z_{\alpha}$, there exists a convergent subsequence $\left\{p_{m_{\imath}}\right\}$ of $\left\{p_{m}\right\}$. We then define $\left\{x_{m}^{(\beta)}\right\}$ to be the sequence $\left\{x_{m_{2}}^{(\alpha)}\right\}$.

Case 2. $\beta$ is a limit ordinal. Then there exists a strictly increasing sequence $\left\{\alpha_{n}\right\}$ of ordinal numbers such that $\alpha_{n}<\beta$ for each $n$ and $\alpha_{n} \rightarrow \beta$, i.e. for every $\alpha<\beta$, there exists $n$ such that $\alpha<\alpha_{n}<\beta$. By dropping a finite number of terms if necessary, we may assume that $\left\{x_{m}^{\left(\alpha_{n}\right)}\right\}$ is a subsequence of $\left\{x_{m}^{\left(\alpha_{p}\right)}\right\}$ whenever $p<n$. We then define $\left\{x_{m}^{(\beta)}\right\}$ to be the sequence constructed from $\left\{x_{m}^{\left(\alpha_{n}\right)}\right\}$ by the diagonal process, i.e., $\left\{x_{m}^{(\beta)}\right\}=\left\{x_{m}^{\left(\alpha_{m}\right)}\right\}$. Then $\left\{x_{m}^{(\beta)}\right\}$ is an essential subsequence of $\left\{x_{m}^{\left(\alpha_{n}\right)}\right\}$ for each $n$. Since $\alpha_{n} \rightarrow \beta,\left\{x_{m}^{(\beta)}\right\}$ is an essential subsequence of $\left\{x_{m}^{(\alpha)}\right\}$ whenever $\alpha<\beta$.

Hence $\left\{x_{m}^{(\alpha)}\right\}$ are defined for all $\alpha<\Omega$. Now for each $\alpha<\Omega$, we let $r_{\alpha}$ be the asymptotic radius of $\left\{x_{m}^{(\alpha)}\right\}$ in $C$. Since $\left\{x_{m}^{(\gamma)}\right\}$ is an essential subsequence of $\left\{x_{m}^{(\delta)}\right\}$ whenever $\delta<\gamma$, and since $r_{\alpha} \geqq 0$ for every $\alpha<\Omega$, the transfinite sequence $\left\{r_{\alpha}: \alpha<\Omega\right\}$ on the real line is decreasing and has lower bound 0 . Let $s=\inf \left\{r_{\alpha}: \alpha<\Omega\right\}$. Then clearly $\lim \left\{r_{\alpha}: \alpha<\Omega\right\}$ exists and equals $s$. This can happen only if for some $\beta_{0}<\Omega, r_{\alpha}=s$ for all $\alpha$ with $\beta_{0}<\alpha<\Omega$. Let 
$\alpha$ be a fixed ordinal with $\beta_{0}<\alpha<\Omega$. We shall show that the asymptotic center $z_{\alpha}$ of $\left\{x_{m}^{(\alpha)}\right\}$ is a fixed point of $T$.

From the way that $\left\{x_{m}^{(\alpha+1)}\right\}$ is constructed from $\left\{x_{m}^{(\alpha)}\right\}$, there exists a convergent sequence $\left\{p_{m}\right\} \subseteq T z_{\alpha}$ with $\lim p_{m}=p \in T z_{\alpha}$ such that

$$
\left\|p_{m}-y_{m}^{(\alpha+1)}\right\| \leqq\left\|z_{\alpha}-x_{m}^{(\alpha+1)}\right\|
$$

for all $m$, where $y_{m}^{(\alpha+1)}=i\left(x_{m}^{(\alpha+1)}\right)$. Since $\left\{x_{m}^{(\alpha+1)}\right\}$ is a subsequence of $\left\{x_{m}^{(\alpha)}\right\}$, and $x_{m}^{(\alpha+1)}-y_{m}^{(\alpha+1)} \rightarrow 0$, we have from (1):

$$
\begin{aligned}
\underset{m}{\lim \sup \left\|p-x_{m}^{(\alpha+1)}\right\|} & =\limsup _{m}\left\|p-y_{m}^{(\alpha+1)}\right\| \\
& \leqq \limsup _{m}\left\|z_{\alpha}-x_{m}^{(\alpha+1)}\right\| \\
& \leqq \lim \sup _{m}\left\|z_{\alpha}-x_{m}^{(\alpha)}\right\|=r_{\alpha}=r_{\alpha+1} .
\end{aligned}
$$

$m$

It follows from the uniqueness of the asymptotic center that $p=z_{\alpha+1}$ and $z_{\alpha}=z_{\alpha+1}$, where $z_{\alpha+1}$ is the asymptotic center of $\left\{x_{m}^{(\alpha+1)}\right\}$ in $C$. Hence $z_{\alpha}=$ $p \in T z_{\alpha}$, completing the proof.

REMARK. Theorem 1 remains true if $X$ is required only to be reflexive and uniformly convex in every direction [6], [3], since in such spaces the asymptotic center of a bounded sequence in a closed convex set is unique [10].

We do not know whether Theorem 1 is true when $C$ is required only to be weakly compact and to have normal structure. For the application of asymptotic center under this setting, see [8] and [9].

\section{REFERENCES}

1. N. A. Assad and W. A. Kirk, Fixed point theorems for set-valued mappings of contractive type, Pacific J. Math. 43 (1972), 553-562.

2. F. E. Browder, Nonlinear operators and nonlinear equations of evolution in Banach spaces, Proc. Sympos. Pure Math., vol. 18, part II, Amer. Math. Soc., Providence, R.I. (to appear).

3. M. M. Day, R. C James and S. Swaminathan, Normed linear spaces that are uniformly convex in every direction, Canad. J. Math. 23 (1971), 1051-1059. MR 44 \#4492.

4. M. Edelstein, The construction of an asymptotic center with a fixed point property, Bull. Amer. Math. Soc. 78 (1972), 206-208. MR 45 \#1005.

5. - Fixed point theorems in uniformly convex Banach spaces (to appear).

6. A. L. Garkavi, The best possible net and the best possible cross-section of a set in a normed space, Izv. Akad. Nauk SSSR Ser. Mat. 26 (1962), 87-106; English transl., Amer. Math. Soc. Transl. (2) 39 (1964), 111-132. MR 25 \#429.

7. E. Lami Dozo, Multivalued nonexpansive mappings and Opial's condition, Proc. Amer. Math. Soc. 38 (1973), 286-292. MR 46 \#9816. 
8. T. C. Lim, A fixed point theorem for families of nonexpansive mappings, Pacific J. Math. (to appear).

9. - - Characterizations of normal structure, Proc. Amer. Math. Soc. 43 (1974), 313-319.

10. - On asymptotic center and its applications to fixed point theory (submitted). 11. J. T. Markin, A fixed point theorem for set valued mappings, Bull. Amer. Math. Soc. 74 (1968), 639-640. MR 37 \#3409.

12. S. B. Nadler, Jr., Multi-valued contraction mappings, Pacific J. Math. 30 (1969), 475-488. MR 40 \#8035.

13. W. Sierpiński, Cardinal and ordinal numbers, 2nd rev. ed., Monografie Mat., vol. 34, PWN, Warsaw, 1965, p. 382 and p. 390. MR 33 \#2549.

Department of Mathematics, Dalhousie University, Halifax, Nova Scotia, CANADA

Current address: Department of Mathematics, University of Chicago, Chicago, Illinois 60637 